\title{
Vasculogenic Mimicry of Oral Malignant Melanoma
}

\author{
You-jin Lee ${ }^{1)}$, Rosario Santos Rivera ${ }^{1)}$, Hitoshi Nagatsuka ${ }^{1)}$, Chong-Huat Siar ${ }^{2)}$, Hg Kok Han ${ }^{3)}$, \\ Eiji Yamagata ${ }^{4}$, and Noriyuki Nagai ${ }^{1)}$
}

\author{
1) Okayama University, Graduate School of Medicine, Dentistry and Pharmaceutical Sciences, Department of Oral Pathology and \\ Medicine, Okayama University, Okayama, Japan. \\ 2) Malaya University, Department of Oral Pathology, Malaysia. \\ 3) Institute for Medical Research, Malaysia \\ 4) Japan Institute for Advanced Dentistry, Osaka.
}

\section{Introduction}

Vasculogenesis is the mechanism of differentiation of precursor cells to endothelial cells that develop de novo vascular networks. We sought to determine this by the use of periodic acid-Schiff (PAS) stain, endothelial markers (CD34, CD105) and laminin, and by transmission electron microscopy in two cases. The results demonstrated that endothelium-lined vessels dominated the tumor microvasculature and these stained positively for PAS, laminin, and endothelial markers. Mosaic and tumorlined vessels were infrequently encountered. Most PAS-positive patterned networks and loops ultrastructurally represented intratumor microhemorrhages that probably arose secondary to tumor vessel leakiness. Vascular channels of the vasculogenic mimicry type were rare. They stained for laminin but not for endothelial markers.

\section{Materials and Methods}

Tissue Specimens from the series of eight cases of primary oral malignant melanoma diagnosed at the Department of Oral Pathology and Medicine, Okayama University, two cases representing the most recently diagnosed (1998 and 1999) were selected for this preliminary study. This is to minimize the inherent problems of preservation of formalin-fixed, paraffin-embedded tissue blocks that may compromise optimal immunohistochemical staining. These slides were reviewed and confirmed as primary oral malignant melanomas. New $4-5 \mu$ m sections were cut and stained routinely with hematoxylin-eosin and periodic acid-Schiff (PAS) without hematoxylin counterstaining so as to reduce background cellular details that may mask the detection of PASpositive patterned networks.

\section{Results}

Light Microscopic and Immunohistochemical Observations Microscopically, both tumors consisted of solid sheets of melanoma cells disposed in a predominant lobular growth pattern. Melanin pigmentation was patchy and of a moderate amount. Except for one case in which the surface oral mucosa was ulcerated and covered with a fibrinopurulent membrane, necrosis was not observed in the multiple tumor samples examined. Hematoxylineosin-stained sections showed tumor microvasculature consisting of scattered irregularly shaped blood vessels and many slit-like channels also containing blood or fibrinous material (data not shown). PAS staining demonstrated the formation of complex networks and closed loops within the vertical growth phase of these tumors, each loop surrounding smaller lobules or nests of melanoma tumor cells (Fig 1A). Immunohistochemical examination showed that some intratumor vessels were positively stained with pan-endothelial marker anti-CD34 antibodies (Fig 1D) but were not positively stained with anti-CD105 antibodies, indicating that these represented normal vessels entrapped within tumor tissues. Other intratumor blood vessels stained positively for both anti- CD34 and anti-CD105 antibodies but with strong expression of the latter marker (Fig 1D,E), thus indicating that these represented areas of activated endothelial cells and tumorassociated neoangiogenesis. Moreover, CD105 stained melanoma cells, suggesting that activated melanoma cells may potentially show endothelial cell-like characteristics, which results in vasculogenic mimicry. However, in areas of PAS-positive vascular loops and patterned networks, a weak to negative reaction for these endothelial markers was observed. Laminin regularly stained the basal lamina of the normal vessels, intratumor blood vessels, and areas containing PAS-positive networks and loops (data not shown). In the latter, laminin positivity produced a reticular meshwork pattern.

\section{Transmission Electron Microscopic Observations}

Tumor-associated vessels, consisting of capillary-like structures lined by normal endothelial cells, were identified within the tumor mass. These probably represented entrapped normal vessels and/ or new tumor vessels co-opted from the existing host vasculature. In the PAS-positive networks and loops, many of these loops were hollow irregular channels, containing red blood cells and fibrinous material and lined with melanoma tumor cells (Fig.2). Most were intratumor microhemorrhages that probably occurred secondary to tumor vessel leakiness. Residual collagen fibrils were identified within them, confirming that these were stromal compartments and not vascular lumens (data not shown). True tumor-lined vessels and mosaic vessels in which endothelial and melanoma tumor cells formed the luminal surfaces were infrequently encountered. Vascular channels composed of solid or hollow ECM columns were also rare. In these channels, red blood cells and an internal basal lamina lining were identified. Melanoma cells lined these channels externally. Endothelium was absent.

\section{Discussion}

Angiogenesis is an essential process in the progression of malignant tumors during growth and metastasis. In these cases, the tumors presented clinically as large pigmented nodular masses arising from the mucoperiosteum of the hard palate and maxillary gingival tissues. Extensive bilateral cervical nodal metastases were present in one case but these tissues were not included for evaluation here. In conclusion, the results of this preliminary study suggest that primary oral malignant melanoma has a heterogeneous tumor microvasculature consisting predominantly of endotheliumlined blood vessels and, to a lesser extent, tumor cell-lined blood vessels and rare matrix-rich vascular channels. 

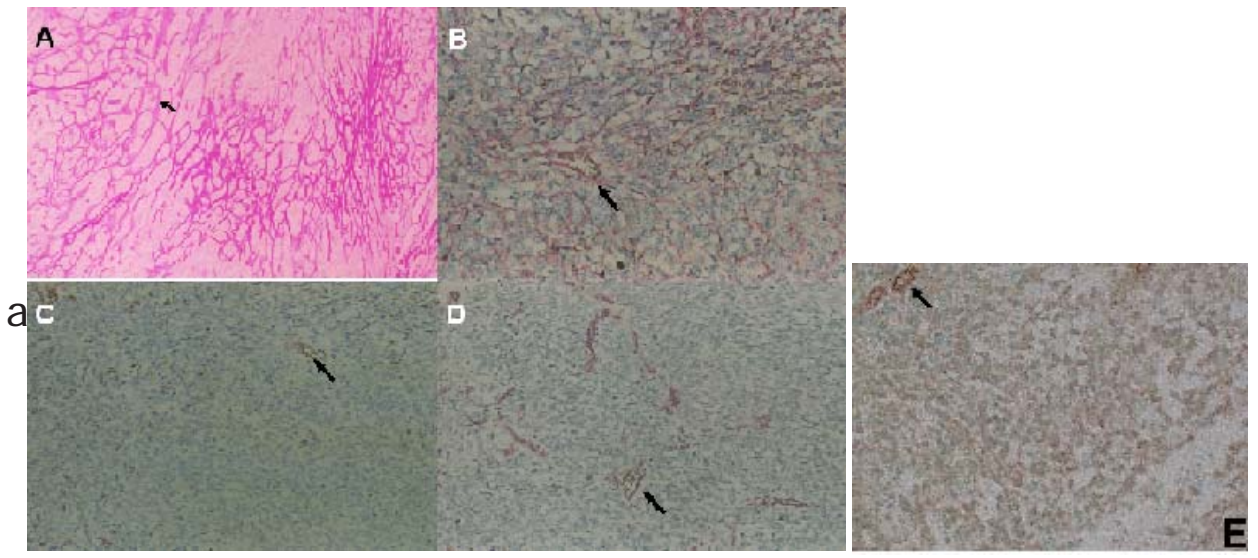

Fig 1a. Light microscopic observations of primary oral malignant melanoma showing a predominant lobular growth pattern, irregularly shaped blood vessels and many slit-like channels(data not shown), formation of complex networks and closed loops (A,B), each loop surrounding smallnests of tumor cells and connection to intratumoral vessels (A,B,C,D arrows). (A) PAS; (B) Type IV collagen; (C)CD31; (D)CD34; (E)CD105 Magnifications: A,C,D 250; B 300; E 350

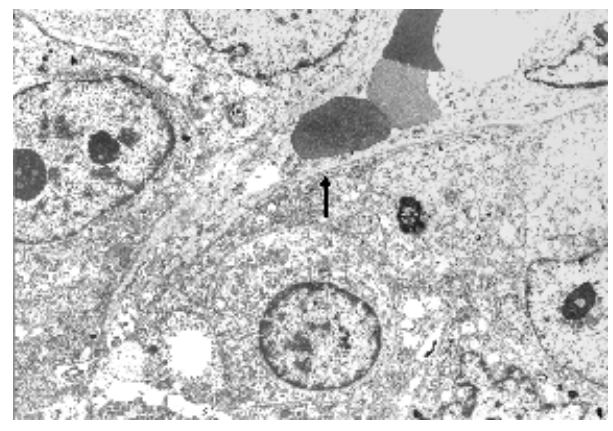

Fig 2. Ultrastructure of vessel-like space with extravasated erythrocytes and fibrinous materials present in the interstices of the melanoma tumor due to intratumor hemorrhage (arrows). Collagen fibrils were identified in these spaces at a higher magnification (not shown), confirming that these spaces are stromal compartments. Magnification 2100.

\section{Reference}

1. Sauter B, Foedinger D, Sterniczky B, Wolff K, Rappersberger K. Immunoelectron microscopic characterization of human dermal lymphatic microvascular endothelial cells: differential expression of CD31, CD34, and type IV collagen with lymphatic endothelial cells vs blood capillary endothelial cells in normal human skin, lymphangioma, and hemangioma in situ. J Histochem Cytochem 46:165-176, 1998

2. Shubik P, Warren BA. Additional literature on "vasculogenic mimicry” not cited. Am J Pathol 156:736, 2000

3. Chang YS, di Tomaso E, McDonald DM, Jones R, Jain RK . Mosaic blood vessels in tumors: frequency of cancer cells in contact with flowing blood. Proc Natl Acad Sci USA 97:14608-14613, 2000

4. Hicks MJ, Flaitz CM. Oral mucosal melanoma: epidemiology and pathobiology. Oral Oncol 36:152-169, 2000

5. Tanaka F, Otake Y, Yanagihara K, Kawano Y, Miyahara R, Li M, Yamada T, et al. Evaluation of angiogenesis in non-small lung cancer: comparison between anti-CD34 antibody and anti-CD105 antibody. Clin Cancer Res 7:3410-3415, 2001

6. Fausto N. Vasculogenic mimicry in tumors. Fact or artifact? Am J Pathol 156:359, 2000

7. Fina L, Moolgard HV, Robertson D, Bradley NJ, Monaghan P, Delia D, Sutherland RD, et al. Expression of CD34 gene in vascular endothelial cells. Blood 75:2417-2426, 1990

8. Clarijs R, Ruiter DJ, de Waal RM. Lymphangiogenesis in malignant tumours: does it occur? J Pathol 193:143-146, 2001

9. Carmeliet P. Mechanisms of angiogenesis and arteriogenesis. Nature Med 6:389-395, 2000

10. Folkman J. Clinical applications of research on angiogenesis. N Engl J Med 333:1757-1763, 1995 